\title{
MOTIVAÇÃO E ESTRATÉGIAS DE APRENDIZAGEM SEGUNDO A TEORIA DAS ABORDAGENS À APRENDIZAGEM: IMPLICAÇÕES PARA A PRÁTICA DE ENSINO-APRENDIZAGEM
}

\author{
MOTIVACIÓN Y EL APRENDIZAJE ESTRATEGIAS ACORDES A LA TEORÍA \\ DE ENFOQUES DE APRENDIZAJE: IMPLICACIONES PARA LA ENSEÑANZA \\ APRENDIZAJE PRÁCTICA
}
MOTIVATION AND LEARNING STRATEGIES ACCORDING TO THE THEORY OF LEARNING APPROACHES: IMPLICATIONS FOR TEACHING-LEARNING PRACTICE

RESUMO: Este artigo tem como objetivo realizar reflexões sobre motivação e estratégias de aprendizagem segundo a teoria das abordagens à aprendizagem de modo a promover melhorias nas práticas de ensino-aprendizagem, uma vez que tanto a motivação como as estratégias utilizadas na aprendizagem possuem relação com o rendimento escolar e qualidade na aprendizagem. Foi possível observar que as motivações e as estratégias de aprendizagem possuem relações entre si dando origem às abordagens à aprendizagem, com o nível de reflexão que o estudante realiza sobre a sua maneira de aprender, podendo ser alteradas de acordo com o nível de conscientização que este estudante possui sobre o tipo de motivação e estratégia de aprendizagem utilizada e/ou por intervenção externa que propicie esta reflexão.

PALAVRAS-CHAVE: Motivação para aprendizagem. Estratégias de aprendizagem. Abordagens à aprendizagem.

RESUMEN: En este artículo se pretende realizar reflexiones sobre las estrategias de motivación y el aprendizaje de acuerdo con la teoría de enfoques de aprendizaje con el fin de promover mejoras en las prácticas de enseñanza y aprendizaje, ya que tanto la motivación y las estrategias utilizadas en el aprendizaje están relacionados con el rendimiento escolar y la calidad en el aprendizaje. Se observó que las motivaciones y estrategias de aprendizaje tienen relaciones entre sí dando lugar a enfoques de aprendizaje, con el nivel de reflexión que el estudiante realiza en su forma de aprender y se puede cambiar de acuerdo con el nivel de conciencia de que esta estudiante tiene sobre el tipo de motivación y el aprendizaje estrategia utilizada y / o mediante la intervención externa que ofrece esta reflexión.

PALABRAS CLAVE: Motivación para el aprendizaje. Estrategias de aprendizaje. Métodos de aprendizaje

\footnotetext{
${ }^{1}$ Instituto Federal de Educação, Ciência e Tecnologia do Rio de Janeiro (IFRJ) e Faculdade de Psicologia
} da Universidade de Lisboa, bolsistas CAPES. 
ABSTRACT: This article aims to conduct reflections about motivation and learning strategies according to the theory of approaches to learning in order to promote improvements in teaching and learning practices, since both the motivation and the strategies used in learning are related to school performance and learning quality. It was possible to verify that the motivations and learning strategies are related with each other giving origin to the approaches to learning, with the level of reflection that the student performs on his way of learning and can be changed according to the awareness level that this student has about the kind of motivation and learning strategy used and / or by external intervention that provides this reflection.

KEYWORDS: Learning Motivation. Learning Strategies. Approaches to learning.

\section{Introdução}

Aprendizagem é um processo complexo que envolve a combinação da motivação do indivíduo para estudar e as estratégias utilizadas por ele para operacionalizar este objetivo (DUARTE et al., 2015). O sucesso e a qualidade dos seus resultados da aprendizagem são dependentes de fatores como os cognitivos, afetivos, interpessoais, escolares, familiares e sociais, sendo necessário identificar e compreender tais fatores para alcançarmos a sua melhoria (DUARTE, 2012). Neste artigo utilizamos o conceito de aprendizagem na perspectiva das abordagens à aprendizagem (Students' Approacches to Learning - SAL) e objetivamos realizar reflexões, por meio de uma revisão de literatura, sobre motivação e estratégias de aprendizagem segundo esta teoria, de modo a promover melhorias nas práticas de ensino-aprendizagem, uma vez que tanto a motivação como as estratégias utilizadas na aprendizagem possuem relação com a qualidade da aprendizagem (DUARTE, 2012).

\section{Aprendizagem dos Estudantes na Perspectiva da Teoria das Abordagens à Aprendizagem ("Students' Approacches to Learning - SAL)}

A linha de investigação SAL (Students Approaches to Learning), sobre a qual esta revisão de literatura está pautada, foi constituída por um grupo de pesquisadores que inaugurou uma nova metodologia e conceito na forma de abordar o estudo da aprendizagem. Seguem uma metodologia de investigação, denominada por Marton (1981) de segunda ordem, dado que os investigadores partiam da perspectiva do aluno na análise da realidade, ou seja, como o estudante percepcionava a aprendizagem 
(LOURENÇO; PAIVA, 2015). Esta metodologia permite-nos conhecer como os estudantes lidam com a aprendizagem assim como a representam. Consequentemente, desta forma é possível pensar em formas de trazer à consciência destes estudantes não só a representação e atuação que fazem da aprendizagem, mas apresentar novas formas de se pensar o ato de aprender assim como as formas práticas de alterá-lo e torná-lo mais rico. Do ponto de vista desta linha de investigação, as abordagens à aprendizagem são de natureza multidimensional (possuem diversas dimensões), tendo significado dentro de um contexto e englobando as intenções dos estudantes em relação ao contexto particular de ensino e aprendizagem onde surgem, bem como com a qualidade dos resultados de aprendizagem (BIGGS, 1993b, apud. LOURENÇO; PAIVA, 2015).

A sustentação desta teoria pauta-se no fato de estar assentada na noção de níveis de aprendizagem; de procurar compreender os processos de aprendizagem em termos de uma associação entre cognição e motivação (sinergia afetiva e cognitva); de apresentarse sólida em termos teóricos, validade e garantia de medidas; poder ser alargada para além da Psicologia da Educação, como por exemplo, para a Psicologia Geral; ser uma possibilidade de solução para a "crise" da aprendizagem (diferentes abordagens conduzem a diferentes níveis de qualidade do produto de aprendizagem; de ter uma perspectiva dominante no que diz respeito à investigação da aprendizagem (DUARTE, 2012).

\section{O Conceito de Abordagens à Aprendizagem}

A abordagem à aprendizagem trata-se de um constructo e relaciona-se à forma como cada estudante interage com a aprendizagem, compreendendo uma componente motivacional (i.e. o que motiva para a aprendizagem) e uma componente estratégica (i.e. que procedimentos de aprendizagem são utilizados) (BIGGS, 1987; MARTON; SÄLJÖ, 2005; RICHARDSON, 2015). A origem do conceito de abordagem à aprendizagem se deu no estudo de Marton \& Säljö em 1976 segundo o qual, investigaram a forma como estudantes abordavam a tarefa de leitura de um texto geral (MARTON; SÄLJÖ, 1976 apud. MARTON; SÄLJÖ, 2005; ENTWISTLE, 2015). Concluíram que há diferenças qualitativas no resultado da aprendizagem: alunos diferentes aprendem aspectos diferentes a partir de um único e mesmo texto e seu 
conhecimento sobre vários princípios científicos, métodos e ideias varia em relação ao que é aprendido em vez de apenas diferir no quanto é aprendido. É uma questão quantitativa, mas principalmente qualitativa. Percebem que alguns estudantes entendem o texto de forma literal, apenas reproduzindo-o. E outros estudantes compreendem o significado do texto no sentido da intenção que o autor tem ao redigi-lo.

Sendo assim, dividem a abordagem em duas dimensões (VALADAS; GONÇALVES; FAÍSCA, 2011): uma abordagem de superfície (corresponde ao primeiro grupo de estudantes e conjuga uma motivação instrumental com uma estratégia de superfície) e uma abordagem profunda (corresponde ao segundo grupo de estudantes e conjuga uma motivação intrínseca com uma estratégia de profundidade).

\section{Motivação e Estratégias de Aprendizagem}

Uma das formas de definir o termo motivação é enquanto estado interno que ativa, orienta e mantém o comportamento, composto por cinco dimensões: a do conteúdo do comportamento, a da velocidade da iniciação deste comportamento, a da intensidade do envolvimento, a da experiência cognitiva e emocional durante o envolvimento e a do que causa o comportamento (DUARTE, 2002). Divide-se em instrumental, intrínseca e de realização.

A motivação instrumental envolve "o desejo de evitar o fracasso através duma correspondência mínima às exigências" (GIBBS apud DUARTE, 2000, p. 34). Implica em avaliar o conteúdo da aprendizagem em questão como desinteressante, com pouca ou nenhuma identificação por parte do estudante, sem relação aos interesses pessoais do mesmo e imposto exteriormente. É considerada um tipo de motivação extrínseca. A motivação intrínseca trata-se do envolvimento direto do estudante com a atividade de aprendizagem já que resulta da satisfação em relação ao próprio conteúdo da aprendizagem. Implica em avaliar o conteúdo da aprendizagem como forma de atualização de interesses ou competências de desenvolvimento pessoal (DUARTE, 2012). A motivação de realização tem um caráter extrínseco, assim como a motivação superficial, mas neste caso, o que mobiliza o estudante é a obtenção de classificações elevadas. O conteúdo da aprendizagem não é o foco da motivação, mas a necessidade de 
fortalecer o seu autoconceito acadêmico através da exibição da excelência pessoal, baseada na competição (DUARTE, 2012).

O termo estratégia de aprendizagem é utilizado conforme Duarte (2000), para designar os meios utilizados pelos indivíduos para se confrontarem com as tarefas de aprendizagem, no que diz respeito ao processamento e utilização da informação. Segundo Biggs (1987), há três níveis de estratégias: micro-estratégias (procedimentos básicos utilizados diretamente nos estudos tendendo a ser particulares às tarefas específicas de aprendizagem); meso-estratégias (estilo de utilização das microestratégias podendo ser dividida em estratégia de superfície, de profundidade e de sucesso); macro-estratégias (processos de auto-regulação das estratégias de aprendizagem que controlam as micro-estratégias e meso- estratégias). Vale lembrar que nas meso-estratégias centra-se a teoria das abordagens à aprendizagem.

As meso-estratégias dividem-se em de superfície e de profundidade como citado no parágrafo anterior. A estratégia de superfície envolve o comportamento de capturar e acumular a informação transmitida para posteriormente ser reproduzida ipsis litteris, podendo, portanto, ser considerada um tipo de estratégia passiva. Os conteúdos são aprendidos de forma sequencial e separada e ao invés de integrados ao conhecimento anterior ou com o conteúdo global daquilo que está sendo estudado. A atenção é focada no símbolo ao invés do significado (DUARTE, 2000). A estratégia de profundidade pode ser considerada uma estratégia ativa, uma vez que, ao contrário da superficial, confronta ativamente as tarefas acadêmicas. O comportamento utilizado neste tipo de estratégia é o de procurar compreender e analisar criticamente o significado do que está sendo aprendido seja entre suas partes como de maneira global, além de poder relacioná-lo com outros assuntos e conhecimentos adquiridos em outras circunstâncias, momentos e de fenômenos reais. A atenção é focada no significado da aprendizagem em questão. "Envolve não apenas a retenção de informação por compreensão, como a formação de um ponto de vista crítico e subjectivo sobre ela, assim como a criação de informação nova (por exemplo, na forma de hipóteses)" (DUARTE, 2000, p. 38). A estratégia de sucesso trata de uma "organização disciplinada das condições materiais da aprendizagem, refletida na gestão estruturada do contexto temporal do estudo" (DUARTE, 2000, p. 39). No uso deste tipo de estratégia, ocorre uma investigação $a$ priori do que é necessário para alcançar uma avaliação de sucesso. A atenção cai sobre os critérios e conteúdos da avaliação, podendo ser denominada por alguns autores, de utilização oportunista quer da estratégia de superfície quer da de profundidade 
(DUARTE, 2000). Ou seja, neste tipo de estratégia pode-se utilizar tanto a estratégia de superfície como a de profundidade dependendo do que for mais conveniente para obterse uma classificação mais elevada em uma avaliação.

Portanto, segundo as classificações explicitadas acima, temos dois tipos principais de motivação e dois tipos principais de estratégias de aprendizagem e outras decorrentes destas combinações.

\section{Tipos de Abordagens à Aprendizagem}

Conforme explicitado, existem dois tipos de abordagens básicas (de superfície e de profundidade) e outras que foram descobertas a partir das duas primeiras em estudos em diferentes contextos.

$\mathrm{Na}$ abordagem de superfície (que compreende um tipo de motivação instrumental e uma estratégia de superfície), o estudante procura corresponder ao mínimo necessário de uma aprendizagem por meio da memorização e reprodução da informação, sem uma preocupação em relacionar o conteúdo aprendido com outros entre si. Lida com o conteúdo de maneira superficial (VALADAS; GONÇALVES; FAÍSCA, 2011; ENTWISTLE, 2015; RICHARDSON, 2015).

$\mathrm{Na}$ abordagem de profundidade (que compreende um tipo de motivação intrínseca e uma estratégia de profundidade), o estudante procura extrair prazer da aprendizagem por meio da compreensão e aprofundamento dos conteúdos a serem aprendidos. Procura relacionar as partes deste conteúdo entre si e com outras, assim como com situações extraescolares. O estudante compreende o significado do que aprende e extrai a mensagem global envolvida com o conteúdo aprendido (DUARTE, 2012).

Segundo Lourenço \& Paiva (2015), as abordagens de superfície e de profundidade foram identificadas em estudantes de diferentes níveis de ensino, na Austrália (DALL’ALBA, 1986), Grã-Bretanha (RAMSDEN, 1981), Holanda (VAN ROSSUM; SCHENK, 1984), Portugal (PAIVA, 2007; ROSÁRIO, 1999) e Espanha (NÚÑEZ; SUÁREZ; CEREZO; GONZÁLEZ-PIENDA; ROSÁRIO; MOURÃO; VALLE, 2013) revelando que elas possuem uma plasticidade, adaptando-se a um conjunto amplo de atividades, particularmente à leitura e escrita, resolução de 
problemas e realização de experiências científicas nas diferentes culturas. Em estudos realizados no Brasil (GOMES, 2010, 2013) com estudantes de Ensino Fundamental e Médio estes resultados também foram encontrados.

Há uma terceira abordagem denominada abordagem de sucesso (BIGGS, 1987) ou abordagem estratégica (ENTWISTLE, 2009, 2015), caracterizada pela conjugação entre uma motivação de realização e uma estratégia de sucesso/organização. Biggs (1987) chegou a esta conclusão por meio de investigações quantitativas e dos instrumentos que criou, o Study Process Questionaire (SPQ) (BIGGS, 1987b) e o Learning Process Questionaire (LPQ) (BIGGS, 1987a) ambos destinados a estudar as abordagens à aprendizagem. Na abordagem de sucesso ou estratégica (ENTWISTLE, 2015), o estudante procura alcançar as melhores classificações nas avaliações por meio de uma organização sistemática do estudo, de modo a corresponder ao máximo o que é solicitado nas avaliações.

Segundo Lourenço e Paiva (2015) posteriormente houve estudos que indicaram hipoteticamente novas características das abordagens apresentadas anteriormente, assim como uma nova abordagem que combina aspectos das abordagens superficial e profunda, denominada de abordagem intermédia. Esta abordagem foi verificada em pesquisas realizadas com estudantes orientais que utilizam de maneira alternada na aprendizagem de um mesmo conteúdo, a memorização e a compreensão. Dessa forma, aquilo que é compreendido passa a ser memorizado. Lourenço e Paiva (2015) afirmam que há autores que subdividem a abordagem intermédia em duas, variando conforme a ênfase que precisam dar à memorização ou à compreensão, precedendo aquela que for mais importante para aquele determinado momento de um mesmo conteúdo estudado.

Duarte (2002) conclui que a maior parte dos estudantes europeus tende a optar alternativamente por uma abordagem superficial ou abordagem profunda, e mais significativamente os estudantes chineses tendem a utilizar ambas as abordagens simultaneamente. Mais atualmente temos estudos comparativos entre culturas (BOWDENA; ABHAYAWANSA; MANZINB, 2013) que confirmam a conclusão anterior. Esta investigação compara estudantes de língua inglesa (Austrália), estudantes de países asiáticos e estudantes de uma universidade australiana. Os resultados encontrados foram que memorização é comum nos três grupos; os estudantes asiáticos e os da universidade australiana adotaram mais a abordagem de profundidade do que os estudantes de língua inglesa local; e os estudantes asiáticos tenderam a usar as duas abordagens em simultâneo. 


\section{Algumas Investigações na Literatura SAL}

Podemos dizer que as investigações que fazem parte da literatura SAL estão divididas em três tipos: estudos descritivos de diferenciação entre os tipos de abordagens à aprendizagem e/ou tipo de orientações motivacionais e/ou tipos de estratégias de aprendizagem utilizados; relação das abordagens à aprendizagem com variáveis, predominando as contextuais e individuais; e as de intervenção, ou seja, que procuram relacionar tipos específicos de intervenção seja individual, em métodos de ensino escolares ou de avaliação e o tipo de abordagem à aprendizagem do estudante (DUARTE, 2002).

No que se refere às abordagens à aprendizagem, Paiva (2007) relata que a adoção de uma abordagem à aprendizagem de superfície se encontra estatisticamente associada a rendimentos escolares inferiores. Resultados semelhantes encontramos no Brasil (GOMES, 2010) que conclui que além da correlação positiva entre abordagem de superfície e rendimento escolar inferior, também abordagens de profundidade correlacionam-se positivamente com rendimento escolar mais altos. Segundo Valadas et al. (2014), a maior parte dos estudos atuais possuem esta tendência de relacionar os tipos de abordagem com o rendimento e sucesso acadêmico dos estudantes. Sendo assim, revela (AK, 2008; VALADAS, 2014) que a abordagem de profundidade está ligada a melhores resultados académicos, assim como a abordagem de superfície está ligada a resultados mais baixos e frágeis. Çetin (2015) realizou um estudo onde procurou verificar se há relação entre as abordagens à aprendizagem com a motivação acadêmica, obtendo resultados positivos. Há ainda outras investigações que apresentam relações entre motivação e estratégia de aprendizagem com sucesso escolar dos estudantes (e.g. BRAN, 2014; MONTEIRO; ALMEIDA; FERNANDES, 2012; SINAPUELAS; STACY, 2015). O estudo de Bran (2014), procurou identificar as abordagens à aprendizagem dos estudantes que se preparam para serem professores. Observaram que a adoção de certas práticas como a elaboração de um diário reflexivo e avaliação por pares promoveram o desenvolvimento de uma abordagem profunda de aprendizagem, assim como o uso de estratégias específicas (métodos, técnicas, e formas de organização) de exercer atividades de planejamento, acompanhamento e auto avaliação. Concluíram que para desenvolver uma abordagem de profundidade deve-se enfatizar o treino de alunos para gerirem sua própria aprendizagem com base no alcance 
dos objetivos de aprendizagem e com os procedimentos adequados às suas próprias necessidades de aprendizagem. O estudo de Monteiro, Almeida e Fernandes (2012) objetivou identificar os fatores preditivos de um rendimento acadêmico excelente com estudantes de cursos de Engenharia. Os resultados revelaram que os melhores alunos apresentaram em sua grande maioria uma abordagem de profundidade. Já a pesquisa de Sinapuelas e Stacy (2015) examina as abordagens à aprendizagem adotadas por estudantes do curso de química introdutória em uma universidade pública. O estudo objetiva também saber como estes estudantes se preparam para os exames durante um semestre. Os resultados mostraram que o tipo de abordagem à aprendizagem dos alunos é um preditor significativo da sua nota no exame. A investigação sugere que os apoios de instrução adicionais devem ser desenvolvidos para possibilitar que mais estudantes usem abordagens de aprendizagem que promovam a aprendizagem quando se estuda e se prepara para os exames.

Algumas investigações concluem que as diferenças na qualidade das aprendizagens estão relacionadas com as abordagens à aprendizagem utilizadas, sendo as abordagens de profundidade correlacionadas positivamente com melhor qualidade na aprendizagem e as abordagens de superfície apresentando correlações negativas com a qualidade na aprendizagem (SUN; RICHARDSON, 2015; TSAI; HWANG, 2015).

As características das abordagens à aprendizagem podem variar em função da natureza das situações, como em diferentes áreas disciplinares e em diferentes tarefas de aprendizagem. Em estudos relatados por Duarte (2002) realizados em Universidades de Ciências Físicas e Naturais em comparação com as áreas de Artes e Ciências Sociais, a abordagem superficial na primeira é caracterizada pela memorização ou aplicação passiva de detalhes, técnicas e fórmulas, e na segunda, caracteriza-se pela memorização de detalhes irrelevantes e generalidades simplificando o significado das ideias principais. No mesmo exemplo, considerando a abordagem profunda, nas Universidades de Ciências Físicas e Naturais, pode muitas vezes precisar como pré-requisito, a memorização de detalhes, técnicas e fórmulas que serão necessárias para um posterior relacionamento entre assuntos e partes contidas no assunto em questão estudado. Já para a área das Artes e Ciências Sociais, desde o início do aprendizado, há uma preocupação em relacionar e interpretar o conteúdo a ser aprendido com a experiência pessoal, assim como com a da sociedade e inter-relacionar as partes com o todo.

Para exemplificar os tipos de abordagem variando em função de tarefas, no caso da tarefa de aprendizagem nas aulas, podemos ter uma abordagem superficial no ato de 
registrar apontamentos sem compreendê-los ou fazer alguma reflexão crítica a respeito do assunto. Na mesma situação, podemos ter uma abordagem profunda ao tomar nota do conteúdo de forma criteriosa, compreendendo o que está sendo anotado, além de conseguir fazer relações entre as partes dadas, acrescentar opiniões e respostas às possíveis dúvidas (DUARTE, 2002). Ou seja, não é a atividade em si que revela o tipo de abordagem à aprendizagem utilizada, mas a intenção que há por trás dela de quem a executa e a maneira que ela é realizada. Sendo assim, uma mesma atividade poderá revelar diferentes tipos de abordagem à aprendizagem.

Gibbs citado por Duarte (2000) revela que, de uma forma geral, a abordagem superficial é a mais utilizada em contextos convencionais de estudo, onde não foram tomadas providências para utilizar a abordagem profunda. Ou seja, a abordagem está mais relacionada com o contexto que a produz do que com os estudantes em si (VALADAS et al., 2014) como podemos confirmar em um estudo (SADEGHI,2015) sobre abordagens à aprendizagem em Ensino Superior da Ásia. Nesta investigação há evidências de que as abordagens de profundidade à aprendizagem estão associadas a uma compreensão profunda de material educativo, mas neste caso, não conclusiva em relação ao alto desempenho acadêmico (medido por resultados da avaliação). Isso pode ser porque os métodos de avaliação adotados por instituições de ensino superior nem sempre recompensam a aprendizagem de uma abordagem profunda. Recomenda-se que as instituições de ensino superior na Ásia tenham como objetivo incentivar os alunos a desenvolver uma abordagem profunda à aprendizagem. Isto pode ser obtido através da incorporação de currículos e avaliação de métodos bem organizados que recompensem uma compreensão profunda, tais como, redação, aprendizagem baseada em problemas, a aprendizagem reflexiva e projetos de investigação.

Segundo Duarte (2000) estudos revelam que a maior parte do conhecimento adquirido em contexto escolar constitui em grande parte, "conhecimento inerte - ou seja, conhecimento que não altera as conceções que os indivíduos têm da realidade e que não afeta o seu comportamento (ou seja, conhecimento que não se transfere, tendo servido apenas para "jogar o jogo da avaliação")" (DUARTE, 2000, p.58). Esta informação é importante para que possamos entender que a abordagem à aprendizagem não deve ser considerada algo que faz parte do indivíduo de forma fixa, como um traço de personalidade, por exemplo. "As abordagens à aprendizagem devem ser vistas como categorias conceptuais e não como descrições de estudantes individuais" (DUARTE, 
2000, p.57). Elas podem variar conforme os estudantes relacionam-se com diferentes contextos de aprendizagem (VALADAS et al., 2014).

Cano (2007) conclui que o ambiente familiar dos estudantes também está associado às abordagens que eles adotam nos estudos, no sentido da família oferecer estímulo às discussões de assuntos intelectuais, ou seja, quanto mais este estudante recebe o estímulo da família, mais ela desenvolve uma abordagem de profundidade e quanto menos recebe o estímulo, mais desenvolve uma abordagem de superfície.

Encontramos também um estudo (YERDELEN-DAMAR E AYDIN, 2015) que é conclusivo sobre o paralelismo entre evasão escolar e abordagem de superfície. Os autores tiveram como objetivo investigar as relações entre as abordagens dos alunos do Ensino Médio para o estudo de ciências, percepções de ambiente de aprendizagem em sala de aula e metas de realização. Verificou-se que a evasão foi associada positivamente com as abordagens de superfície para a aprendizagem de ciências.

Segundo Duarte (2012) vários autores apontam que os diferentes níveis de sucesso e qualidade de aprendizagem estão relacionados com as abordagens à aprendizagem e as suas concepções de aprendizagem. "Possivelmente, esta intervenção poderá compreender procedimentos que possibilitem uma consciência metacognitiva sobre as concepções pessoais, o conhecimento de concepções alternativas e a eventual reestruturação das concepções pessoais" (REBELO; DUARTE, 2012). Partindo do princípio que a atuação dos estudantes em termos motivacionais e estratégicos é dirigida pelas suas concepções de aprendizagem, então é válido admitir que o mapeamento conceitual desse fenômeno é prioritário; não apenas para a compreensão dos insucessos acadêmicos, como também para a promoção de mudanças das próprias concepções, tendo como objetivo último, o agenciamento da auto regulação e a conquista de aprendizagens mais ricas (FREIRE; DUARTE, 2010). Marton e Booth (1997) encontraram uma relação de causa e efeito entre as abordagens à aprendizagem utilizadas pelos estudantes com suas concepções de aprendizagem. No entanto, outros estudos (EDMUNDS; RICHARDSON, 2009) verificaram uma baixa correlação entre as variáveis.

Em termos de intervenção, há um estudo (ÇOLAK, 2015) que objetivou determinar a eficácia das atividades de aprendizagem cooperativa no sentido de garantir a aprendizagem profunda de acordo com estilos de aprendizagem dos alunos, concluindo que os alunos com estilos de aprendizagem cooperativos e competitivos 
conseguiram uma abordagem de aprendizagem profunda do que os alunos com estilos de aprendizagem de evitação, dependentes e participativos.

Ainda em termos de intervenção, um estudo (CLINTON, 2014) que objetivou relacionar as abordagens preferidas dos alunos para a aprendizagem e comportamentos durante a aprendizagem onde foi reproduzida a leitura de textos como no modelo de Biggs (1987), foi constatado que a mediação do comportamento durante o processo de aprendizagem explicou a associação positiva entre uma abordagem profunda à aprendizagem e respostas precisas às questões de compreensão.

Atualmente, Entwistle (2015) traça as origens do constructo de abordagens à aprendizagem (motivações e estratégias de aprendizagem) e procura atualizar o tema, esclarecendo como o conceito originado dentro de um clima de investigação que incidiu diretamente sobre a experiência de estudantes, foi evoluindo posteriormente. Esta revisão de pesquisas revelou como este construto está a ser amplamente utilizado, mas segundo ele, não necessariamente está sendo bem entendido, trazendo consequências para as interpretações das investigações. Entwistle (2015) identificou estudos (PROSSER; TRIGWELL; TAYLOR, 1994, apud. ENTWISTLE, 2015), onde as abordagens de ensino de professores são descritas sugerindo um paralelismo com as descrições da aprendizagem dos alunos. Eles descobriram que os professores pareciam concentrar uma restrição sobre a informação a ser transmitida para os alunos em termos de suas próprias maneiras de compreensão da disciplina. Por outro lado, havia estudos que professores relatavam tentar ver o conteúdo do ponto de vista dos alunos e desenvolvimento conceitual de forma a encorajar o conhecimento e aprofundamento dos alunos. Posteriormente, foi encontrada (TRIGWELL; PROSSER; WATERHOUSE, 1999, apud. ENTWISTLE, 2015) diferentes abordagens para ensino para ser correlacionado com o equivalente às abordagens à aprendizagem. Outro grande desenvolvimento da ideia de "abordagens à aprendizagem" ocorreu por meio da ajuda de estudantes para pensar sobre suas próprias formas de estudar e por conceber um currículo destinado a incentivar e apoiar a abordagem mais profunda eficazmente (GIBBS, 1981, apud. ENTWISTLE, 2015). Hattie (2014) descreveu uma pesquisa neurológica que mostra o distintivo processamento ocorrendo em partes diferentes do cérebro, ao efectuar as tarefas concebidas para evocar a aprendizagem de dois diferentes processos como no caso da tarefa de leitura. Contudo, tem-se revelado difícil conceber qualquer forma de incentivar a abordagem profunda, já que as formas específicas de desenvolvimento de uma compreensão dependem crucialmente da natureza do sujeito e 
o contexto em que a aprendizagem ocorre (BAETEN et al., 2010; BIGGS; TANG, 2011; ENTWISTLE, 2009; GIJBELS; SEGERS; STRUYF, 2008, apud. ENTWISTLE, 2015).

Especificamente no contexto brasileiro, as investigações dentro do referencial teórico SAL são escassas, tendo sido encontrados nos últimos anos, somente os estudos de Gomes (2010); Gomes, Golino, Pinheiro, Miranda e Soares (2011); Gomes (2013), Gomes e Golino (2014) e Costa, Pfeuti e Nova (2014), alguns deles já foram citados neste artigo. O estudo de Gomes (2010) procurou investigar relações entre rendimento escolar em grupos de estudantes de Ensino Fundamental e Médio com perfis distintos de abordagens à aprendizagem. O estudo de Gomes, Golino, Pinheiro, Miranda e Soares (2011) objetivou validar a Escala de Abordagens de Aprendizagem (EABAP) (que inclui itens sobre motivação e estratégias para a aprendizagem) em uma Amostra Brasileira de Ensino Fundamental e Médio que foi publicada posteriormente para melhorias de algumas limitações do estudo anterior (Gomes, 2013), tendo como resultados um equilíbrio nos itens que mensuram os modelos de abordagens de superfície e de profundidade, a possibilidade de medir em separado cada abordagem e em função da idade e da série por meio das escalas específicas que o instrumento apresenta e dando condições das escolas planejarem melhor as ações pedagógicas direcionadas à aprendizagem dos estudantes. A investigação de Gomes e Golino (2014) testou as hipóteses de que as percepções autorrelatadas de estudantes sobre seus processos acadêmicos são um tipo de conhecimento metacognitivo acadêmico utilizando três hipóteses sendo que uma delas é baseada nas abordagens à e concluindose que o uso de modelos de predição de aprendizagem representam um componente inovador para as técnicas tradicionais e que pode nos ajudar a compreender as interações entre as variáveis educacionais e psicológicas na predição de resultados académicos, representando um avanço em termos de inferência. E finalmente, a pesquisa de Costa, Pfeuti e Nova (2014) que analisou o impacto da utilização de diferentes estratégias de ensino pelos professores (uma considerada como de superfície e outra como de profundidade), levando-se em conta a forma de estudo que se pretende que os alunos adotem em um curso de pós-graduação em Ciências Contábeis, entre elas as abordagens superficial e profunda de aprendizagem. Dentre os estudos com mais de cinco anos, encontramos um autor (SOBRAL, 2001) que objetivou relacionar a reflexão dos estudantes de medicina em relação a aprendizagem, às abordagens para estudar e 
desempenho acadêmico, havendo correlação positiva e significativa entre as três variáveis.

Em Portugal, há investigações que relacionam abordagens à aprendizagem com sucesso acadêmico em alunos de Ensino Secundário (equivalente ao Ensino Médio) como o de Rosario (1999) que procurou conhecer as estratégias de aprendizagem mais usadas em estudantes de Ensino Médio português e a sua correlação com as classificações escolares, encontrando resultados estatisticamente significativos.

\section{Considerações finais}

Este artigo teve o objetivo de fazer uma reflexão por meio da apresentação de estudos sobre motivação e estratégias de aprendizagem segundo a teoria das abordagens à aprendizagem de modo a promover melhorias nas práticas de ensino-aprendizagem.

Foi possível constatar que tanto a motivação como as estratégias utilizadas na aprendizagem possuem relação com o rendimento escolar, qualidade na aprendizagem, evasão e abandono escolar, concepções de aprendizagem, entre outras.

Observamos também que o nível de consciência e reflexão que o estudante possui sobre a sua maneira de aprender reflete no seu tipo de abordagem de aprendizagem ou seja, na sua motivação para aprender e no tipo de estratégia que utilizada, sendo portanto, possível de alterações. Sendo assim, é possível por meio de intervenção, alterar as abordagens à aprendizagem (motivações e estratégias) trabalhando diretamente o nível de conscientização que este estudante possui sobre o tipo de motivação e estratégia de aprendizagem utilizada e/ou por intervenção indireta, como por exemplo, alteração de currículo, avaliação, que propicie esta mudança.

Sendo assim, nesta abordagem teórica, existe uma contribuição no sentido de que tanto a motivação como a estratégia de aprendizagem pode ser alterada diante de transformações internas do estudante proporcionadas por intervenções que possibilitem auto reflexões sobre o ato de aprender, estimulando uma modificação de dentro para fora, prática pouco usada na maior parte dos projetos que se propõem a intervir no ensino-aprendizagem. 


\section{REFERÊNCIAS}

AK, S. A conceptual analysis on the approaches to learning. Educational Sciences: Theory \& Practice, v. 8, n. 3, p. 707-720, 2008.

BEYAZTAS, D. D.; SENEMOGLU, N. N. Learning approaches of successful students and factors affecting their learning approaches. Education \& Science / EgitimVeBilim, v. 40, n. 179, p. 193-216, 2015.

BRAN, C. B. Strategies for developing a deep approach of learning in higher education. Journal Plus Education / Educatia Plus, v. 11, n. 2, p. 130-140, 2014.

BIGGS, J. Student approaches to learning and studying. Australia: Australian Council for Educational Research Limited, 1987.

BIGGS, J. B. The Learning Process Questionnaire (LPQ): Users' manual. Hawthorn, Australia: Australian Council for Educational Research, 1987a.

BIGGS, J. B. The Study Process Questionnaire (SPQ): Manual. Hawthorn, Australia: Australian Council for Educational Research, 1987b.

BOWDEN, M. P., ABHAYAWANSA, S.; MANZIN, G. A multiple cross-cultural comparison of approaches to learning. Compare: A Journal Of Comparative And International Education, v. 45, n. 2, p. 272-294, 2015.

CANO, F. Approaches to learning and study orchestrations in high school students. European Journal of Psychology of Education, v. 22, n. 2, p. 131-151, 2007.

ÇETIN, B. Predicting academic success from academic motivation and learning approaches in classroom teaching students. Contemporary Issues In Education Research, v. 8, n. 3, p. 171-180, 2015.

CLINTON, V. V. The relationship between students' preferred approaches to learning and behaviors during learning: An examination of the process stage of the 3P model. Instructional Science, v. 42, n. 5, p. 817-837, 2014.

COSTA, S. A., PFEUTI, M. M.,; NOVA, S. P. C. As estratégias de ensinoaprendizagem utilizadas pelos docentes e sua relação com o envolvimento dos alunos. Revista Evidenciação Contábil \& Finanças, v. 2, n. 1, p. 59-74, 2014.

ÇOLAK, E. E. The effect of cooperative learning on the learning approaches of students with different learning styles. Eurasian Journal Of Educational Research (EJER), v. 59, p. 17-34, 2015.

DALL'ALBA, G. Learning strategies and the learner's approach to a problem solving task. Research in Science Education, v. 16, p. 11-20, 1986.

DUARTE, A. M. Avaliação e modificação de concepções, motivações e estratégias de aprendizagem. Tese de doutoramento não publicada. Lisboa: Faculdade de Psicologia e de Ciências da Educação da Universidade de Lisboa, 2000.

DUARTE, A. M. Aprendizagem, Ensino e Aconselhamento Educacional. Porto: 
Porto Editora, 2002.

DUARTE, A. M. Aprender Melhor: aumentar o sucesso e a qualidade da aprendizagem. Lisboa: Escolar Editora, 2012.

DUARTE, A. M., CABRITO, B., FIGUEIRA, A. I.; MONGE, J. Teaching practices for passive and active learning in rural and urban elementary teachers. Sisyphus: Educational Sciences Journal, v. 3, n. 2, p. 134-154, 2015.

EDMUNDS, R., \& RICHARDSON, J. T. E. Conceptions of learning, approaches to studying and personal development in UK higher education. British Journal of Educational Psychology, v. 79, p. 295-309, 2009.

ENTWISTLE, N. J. Teaching for understanding at university: Deep approaches and distinctive ways of thinking. Basingstoke: Palgrave Macmillan, 2009.

ENTWISTLE, N.J. The origins and evolution of the concept of 'approaches to learning' The Psychology of Education Review, v. 39, n. 2, 2015.

FREIRE, G. L., \& DUARTE, A. M. Concepções de aprendizagem em estudantes universitários brasileiros. Psicologia USP, v. 21, n. 4, p. 875-898, 2010.

GOMES, C. M. A. Perfis de estudantes e a relação entre abordagens de aprendizagem e rendimento escolar. Psico, v. 41, n. 4, p. 503-509, 2010.

GOMES, C. M. A., GOLINO, H. F., PINHEIRO, C. A. R., MIRANDA, G. R.; SOARES, J. M. T.Validação da escala de abordagens de aprendizagem (EABAP) em uma amostra brasileira. Psicologia: Reflexão e Crítica, v. 24, n. 1, p. 19-27, 2011.

GOMES, C. M. A. A construção de uma medida em abordagens de aprendizagem. Psico, v. 44, n. 2, p. 193-203, 2013.

GOMES, C. M. A.; GOLINO, H. F. Autorrelatos de estudantes sobre seus processos de aprendizagem são conhecimento metacognitivo acadêmico. Psicologia Reflexão e Crítica, v. 27, n. 3, p. 472-480, 2014.

HATTIE, J. Advancing a science of learning. 34th Vernon-Wall Lecture, presented at the British Psychological Society Psychology of Education Section Annual Conference in Milton Keynes, November, 2014.

LOURENÇO, A. A.; PAIVA, M. O. Abordagens à aprendizagem: a dinâmica para o sucessoacadémico. CES Psicología, v. 8, n. 2, p. 47-75, 2015.

MARTON, F. Phenomenography - describing conceptions of the world around us. Instituctional Science, v. 10, p. 177-200, 1981.

MARTON, F.; BOOTH, S. Learning and awareness. Mahwah: Lawrence Erlbaum, 1997.

MARTON, F.; SALJO, R. Approaches to learning. In Marton, F., Hounsell, D. and Entwistle, N., (eds.) The experience of learning: implications for teaching and studying in higher education. 3rd (Internet) edition (pp. 39-58). Edinburgh: University 
of Edinburgh, Centre for Teaching, Learning and Assessment, 2005.

MONTEIRO, S. C. ALMEIDA, L. S.; VASCONCELOS, R. C. Abordagens à aprendizagem, autorregulação e motivação: convergência no desempenho acadêmico excelente. Revista Brasileira de Orientação Profissional, v. 13, n. 2, p. 153-162, 2012.

NÚÑEZ, J. C., SUÁREZ, N., CEREZO, R., GONZÁLEZ-PIENDA, J., ROSÁRIO, P., MOURÃO, R.; VALLE, A. Homework and academic achievement across Spanish Compulsory Education, Educational Psychology , v. 3 , p. 1 - 21, 2013.

PAIVA, M. O. A. Abordagens à aprendizagem e abordagen são ensino: uma aproximação à dinâmica do aprender no secundário. Tese de doutoramento não publicada. Instituto de Educação, Universidade do Minho, Braga, 2007.

RAMSDEN, P.A. Study of the relationship between student learning and its academic context. Unpublished doctoral dissertation, University of Lancaster, UK (British Theses No DX42179/82AX), 1981.

REBELO, I., \& DUARTE, A. M. Concepções de aprendizagem com o computador em estudantes universitários. Psicologia, v. 26, n. 2, p. 87-111, 2012.

RICHARDSON, J. T. Approaches to learning or levels of processing: what did Marton and Säljö (1976a) really say? the legacy of the work of the Göteborg Group in the 1970s. Interchange: A Quarterly Review Of Education, v. 46, n. 3, p. 239-269, 2015.

ROSÁRIO, P. Variáveis cognitivo-motivacionais na aprendizagem: as "abordagens ao estudo em alunos do Ensino Secundário. Tese de Doutoramento não publicada. Braga: Instituto de Educação e Psicologia da Universidade do Minho, 1999.

SADEGHI, M. R. Learning in higher education, miscellaneous approaches. Modern Journal of Language Teaching Methods, v. 5, n. 1, p. 295-300, 2015.

SINAPUELAS, M. L. S.; Stacy, A. M. The Relationship between student success in introductory university chemistry and approaches to learning outside of the classroom. Journal of Research in Science Teaching, v. 52, n. 6, p. 790-815, 2015.

SOBRAL, D. T. Medical students' reflection in learning in relation to approaches to study and academic achievement. Medical Teacher, v. 23, n. 5, p. 508-513, 2001.

SUN, H.; RICHARDSON, J. T. E. Students' perceptions of the academic environment and approaches to studying in British postgraduate business education. Assessment \& Evaluation in Higher Education, v. 40, n. 6, 2015.

TSAI, P-S, TSAI, C-C.; HWANG, G-H. The effects of instructional methods on students' learning outcomes requiring different cognitive abilities: context-aware ubiquitous learning versus traditional instruction. Interactive Learning Environments, v. 23, n. 3, 2015.

VALADAS, S. S., ARAÚJO; ALMEIDA, L. Abordagens ao estudo e sucesso academico no ensino superior. Revista Eletronica de Psicologia, Educação e Saúde, v. 4, n. 1, p. 47-67, 2014. 
VALADAS, S. S., GONÇALVES, F. R.; FAÍSCA, L. M. Perfis de aprendizagem de estudantes do ensino superior: Abordagens ao estudo, concepções de aprendizagem e preferências por diferentes tipos de ensino. Análise Psicológica, v. 29, n. 3, p. 369-389, 2011.

VAN ROSSUM, E. J.; SCHENK, S. M. The relationship between learning conception, study strategy and learning outcome. British Journal of Educational Psychology, v. 54 , p. 73-83, 1984.

YERDELEN-DAMAR, S.; AYDIN, S. Relations of approaches to learning with perceptions of learning environment and goal orientations. Education \& Science / EgitimVeBilim, v. 40, n. 179), p. 269-293, 2015.

\section{Como referenciar este artigo}

FONTES, Marisa Aguetoni. Motivação e estratégias de aprendizagem segundo a teoria das abordagens à aprendizagem: implicações para a prática de ensino-aprendizagem. Revista Ibero-Americana de Estudos em Educação, Araraquara/SP, v. 11, n. esp. 3, p.1727-1744, 2016. Disponível em: 〈http://dx.doi.org/10.21723/riaee.v11.n.esp3.9081〉. E-ISSN: 1982-5587.

Submetido em: agosto/2016

Aprovado em: novembro/2016 